\title{
Dynamic Fuzzy c-Means (dFCM) Clustering and its Application to Calorimetric Data Reconstruction in High Energy Physics
}

\author{
Radha Pyari Sandhir, Sanjib Muhuri and Tapan K. Nayak \\ Variable Energy Cyclotron Centre, Kolkata - 700064, India
}

\begin{abstract}
In high energy physics experiments, calorimetric data reconstruction requires a suitable clustering technique in order to obtain accurate information about the shower characteristics such as position of the shower and energy deposition. Fuzzy clustering techniques have high potential in this regard, as they assign data points to more than one cluster, thereby acting as a tool to distinguish between overlapping clusters. Fuzzy c-means (FCM) is one such clustering technique that can be applied to calorimetric data reconstruction. However, it has a drawback: it cannot easily identify and distinguish clusters that are not uniformly spread. A version of the FCM algorithm called dynamic fuzzy cmeans (dFCM) allows clusters to be generated and eliminated as required, with the ability to resolve non-uniformly distributed clusters. Both the FCM and AFCM algorithms have been studied and successfully applied to simulated data of a sampling tungsten-silicon calorimeter. It is seen that the FCM technique works reasonably well, and at the same time, the use of the dFCM technique improves the performance.
\end{abstract}

Keywords: Soft computing, Clustering, Fuzzy logic, FCM, Sampling calorimeter

\section{Introduction}

The human mind can easily grasp concepts like imprecision, uncertainty, partial truth and approximation. Conventional (hard) computing, i.e., computing with traditional electronic computations has limitations, as it only sees in terms of 'black' and 'white', or more accurately, zeroes and ones. Though this way of computing is sufficient for a large number of tasks, it cannot handle a problem for which there is not yet enough information to calculate definitively what the answer is. Soft computing, on the other hand, uses nature as a role model, allowing computers to take uncertainty and imprecision into account, in much the same way the human mind does. Soft computing surfaced as a formal computer science area of study [1] in the early 1990's, and acts as the emerging field of 'computational intelligence' essentially adding 'intelligence' to computing techniques [2] . It is an essential tool for a good decision making model, and has been used in a wide variety of disciplines from bioinformatics to aeronautical engineering to image processing. Computational intelligence has also found its way to detector physics, especially for high energy physics experiments, and has been used to determine detector performances [3, 4, 5], as well as extract physical information of interacting particles $[6,7,8,8]$.

In high energy physics experiments, one of the essential steps in the extraction of physical information from particle detectors is to reconstruct the characteristics of the incoming particles. A calorimeter is normally used for accurate characterization of an incoming particle in terms of its position and energy. Depending on the structure, calorimeters can be categorized into two types: homogeneous and sampling. A homogeneous calorimeter is made up of a single block of material that acts as an absorber as well as an active medium from which the signal can be collected. On the other hand, sampling calorimeters are segmented in the longitudinal as well as in the transverse directions, consisting of layers of absorber and active detector combinations. Each segment or cell acts as an independent detector. To reconstruct the physical information one needs to identify the group of hit cells associated with an incoming particle, and determine the most probable position of the particle and its energy. This is done by the use of a clustering algorithm. Additionally, the clustering techniques can also be used to classify different sets of events [10].

A number of clustering algorithms exist in the literature, and can be employed for high energy physics data reconstruction. Algorithms like the contiguity method and cellular automata, search neighborhoods of cells would 
not be as useful for distinguishing between overlapping clusters found in high density environments. Some other methods, like deterministic annealing, require parameters that depend very strongly on the data pattern, and would not serve well as a generic clustering algorithm. Hard clustering techniques such as local maxima search, connectedcell search and k-means clustering simply assign a data point to a cluster. A data point either lies in a cluster or it does not, and so, overlapping clusters are hardly distinguishable. In fuzzy clustering, on the other hand, data points are represented by degrees of membership, i.e., they lie within clusters to varying degrees. The term 'fuzzy' is used because an observation may in fact lie in more than one cluster simultaneously, as is the case with many high energy physics applications. In this article, a type of fuzzy clustering called fuzzy c-means (FCM) [11, 12, 13] is studied and applied to simulated data of a sampling tungsten-silicon calorimeter. A modification to the FCM algorithm [14], that allows the clustering to be carried out dynamically was found to resolve particle clusters well specifically in the case of nonuniformly distributed clusters.

This article is organized as follows. Section 2 discusses the FCM algorithm including possible modifications. One such modification is the dynamic FCM (dFCM) technique, which is discussed in Section 3 . Details of the calorimetric configuration are outlined in Section 4 The power of FCM to distinguish between overlapping clusters is demonstrated in Section 5 by performing clustering on two photon clusters obtained by neutral pions of varying energies. The ability of dFCM to resolve non-uniformly distributed clusters with ease unlike FCM, is demonstrated in Section 6 , followed by its application to calorimetric data. The paper concludes in Section 7 with a summary and a remark on future perspectives.

\section{Fuzzy Clustering and the FCM Algorithm}

Fuzzy clustering is a technique in which the allocation of data points to clusters follows fuzzy logic, thereby providing the means to separate overlapping clusters. Fuzzy clustering is appropriate for applications in detectors, as showers generated by particles may have profiles that are continuous or overlapping on the detector planes. Fuzzy c-means (FCM) is a commonly used fuzzy clustering algorithm [11, 12, 13]. It is a version of the k-means algorithm that incorporates fuzzy logic, so that each point has a weak or strong association to the cluster, determined by the inverse distance to the center of the cluster. The centers obtained using the FCM algorithm are based on the geometric locations of the data points. The FCM algorithm has been used in [15] to track gamma rays in segmented detectors, and in [3] to find clusters in the preshower detector in high energy heavy ion experiments.

For a set of data points $X$, FCM seeks to minimize the objective function - the weighted within groups sum of squared errors $J_{m}$ :

$$
J_{m}(U, V ; X)=\sum_{k=1}^{n} \sum_{i=1}^{C}\left(u_{i k}\right)^{m}\left\|x_{k}-v_{i}\right\|^{2},
$$

where $V=\left(v_{1}, v_{2}, \ldots, v_{C}\right)$ is a vector of unknown cluster centers or centers, $U$ consists of the memberships $u_{i k}$ of the $k^{\text {th }}$ point in the $i^{\text {th }}$ cluster, and $\|x\|=\sqrt{x^{T} x}$ is any inner product norm. The fuzzy factor $m$ normalizes and fuzzifies the memberships so that their sum is 1 . A value of 2 implies linear normalization, whereas when $m$ is closer to 1 , the cluster center closest to the point is given much more weight than the others, making the algorithm similar to k-means. Optimization of $J_{m}$ is based on iteration through certain necessary conditions. Following the FCM Theorem [12] If $D_{i k}=\left\|x_{k}-v_{i}\right\|>0$ for all $i$ and $k$, then $(\mathrm{U}, \mathrm{V})$ may minimize $J_{m}$ only if, when $m>1$,

$$
u_{i k}=\left[\sum_{j=1}^{C}\left(\frac{D_{i k}}{D_{j k}}\right)^{\frac{2}{m-1}}\right]^{-1}
$$

where $1 \leq i \leq C, \quad 1 \leq k \leq n$, and

$$
v_{i}=\frac{\sum_{k=1}^{n}\left(u_{i k}\right)^{m} x_{k}}{\sum_{k=1}^{n}\left(u_{i k}\right)^{m}} .
$$

Alternating optimization (AO) is the iteration technique that is most often used in this algorithm. It simply loops through one cycle of estimates for $V_{t-1} \rightarrow U_{t} \rightarrow V_{t}$ until some error criteria is reached. An error threshold $\epsilon$ can be specified so that the error criteria is $\left\|V_{t-1}-V_{t}\right\|_{e r r} \leq \epsilon$. Defuzzification, that is, determining which point lies in which cluster, can be done by looking at the memberships of a point associated with each cluster. A point belongs to a 
cluster if its corresponding membership is the maximum out of the point's memberships in all of the clusters. Figure 1 shows the flow chart of the FCM algorithm. The algorithm runs for a specified number of clusters. This number can be varied, and the best set of clusters can be selected by means of a validity index.

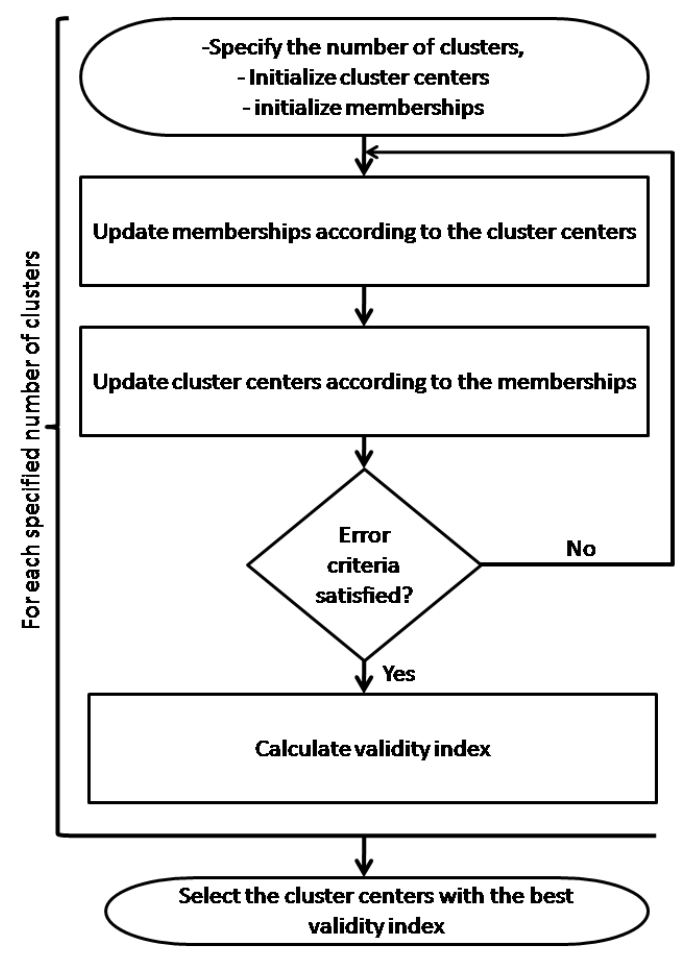

Figure 1: The flow chart for the fuzzy c-means (FCM) algorithm.

\subsection{Validity Indices}

A validity index seeks to determine how well the data is represented by the selected clusters. There are a number of validity indices available in the literature [13]. The Xie-Beni index [16] is one such validity index that is widely used because of its dependence on both memberships as well as geometric distances. More explicitly, the index depends on the distances between data points and the centers of clusters as well as the distance between cluster centers. Other indices are based on other aspects of the data. For instance, the partition coefficient depends only on the memberships of the data:

$$
P C(c)=\frac{1}{n} \sum_{i=1}^{c} \sum_{j=1}^{n} \mu_{i j}^{2},
$$

where $n$ is the number of data points, $c$ is the number of clusters, and $\mu_{i j}$ is the membership of the $j^{\text {th }}$ data point in the $j^{\text {th }}$ cluster. It varies from $1 / c$ to 1 . This index is to be maximized. In order to remove the dependence of $P C$ on $c$, the Modified Partition Coefficient was defined as [17]:

$$
\operatorname{MPC}(c)=1-\frac{c}{c-1}(1-P C)
$$

which varies from 0 to 1 . This index is to be maximized as well. The Xie-Beni index is chosen for the present study, though it may be noted that any validity index preferred by the experimenter may be employed. The Xie-Beni index 
$v_{X B}$ is defined as follows:

$$
v_{X B}(U, V ; X)=\frac{\sum_{i=1}^{C} \sum_{k=1}^{n} u_{i k}^{2}\left\|x_{k}-v_{i}\right\|^{2}}{n(\underbrace{\min }_{i \neq j}\left\{\left\|v_{i}-v_{j}\right\|\right\})} .
$$

It is essentially the ratio of the total variation of the cluster centers and memberships of the observations in the groupings to the separation between the cluster centers, and minimization of the index leads to better clusters. The larger the separation between clusters and the more closely packed the points in the cluster, the better the clustering. This index has no upper bound.

\subsection{Modifications to the FCM Algorithm}

Over the years, a number of application-specific modifications have been made to the FCM algorithm. As FCM clustering is based on the Euclidean distance between data samples, it gives each data point and each dimension (or feature) the same importance. A modification to FCM using feature-weight learning was looked into [18, 19], however, in the present study, each of the three dimensions considered in the clustering should be given equal importance. The drawbacks of FCM include the dependence on the fuzzy factor $m$, which may vary from one data set to other [20], and the fact that it treats outliers in the same way it treats data points lying in the bulk of the data. In order to address these concerns, the suppressed FCM algorithm [21] was looked into. This algorithm prizes the biggest memberships and suppresses the others with a weighting factor.

A modified version of the FCM algorithm [14] has been developed, which has the main advantage that it dynamically finds clusters as data streams in, deleting and generating clusters as needed. The decision making for valid clusters is made through the use of a validity index. Theoretically, the method does not require a maximum number of clusters, only a minimum, which gives it an edge over the energy-weighted modification. It also adapts to the data pattern at each instant. This modified algorithm is called dynamic Fuzzy c-Means (dFCM), which is discussed in detail in the next section.

\section{The dynamic Fuzzy c-Means (dFCM) Algorithm}

The dFCM algorithm [14] is a modification of the fuzzy c-means algorithm, allowing cluster centers to be adaptively updated as data points keep streaming in. If a new cluster is formed, then a new cluster center is automatically generated. Figure 2 gives a detailed flow chart of the algorithm. The working of the algorithm is as follows:

1. To start with, we assume that we already have a few of the incoming data points at hand. From these data points, we can roughly estimate - but not restrict ourselves to - the range of the incoming data. Initially, a few parameters are specified, namely, the membership threshold, $\mu$, the FCM error criteria $\epsilon$, and the bounds of the variable $C$ - the number of clusters. The initial number of points is taken to be low, just to kick-start the clustering and give the algorithm a brief idea about the values it is dealing with. Unless the initial number of points is so large that hints of clusters can already be seen, the clustering wont be affected.

2. If the minimum value of the variable $C$ is $C_{\min }$ then, initially, $C_{\min }$ cluster centers are generated uniformly within the input space. Note that this number must be greater than or equal to 2 as the unit cluster is not allowed in the FCM algorithm. Once the initial cluster centers have been specified, the memberships of the initial data points are found using Equation 2

3. The data points are now allowed to stream in, one by one. When a new data point arrives, its memberships in the clusters present are calculated. If the maximum membership value associated with this point is greater than or equal to the membership threshold $\mu$, then a simple AO update takes place, as outlined in Section 2, This means that the data point belongs to at least one of the clusters to an extent that matches or exceeds $\mu$.

4. Let $C$ be the number of clusters present at a given time. If the maximum membership of the data point falls below $\mu$, then the validity of the present number of cluster centers $C$ is compared with each of the validities of $L=C-2$ to $C+2$ clusters in the following manner:

- The stored values of the cluster centers are checked to see if $L$ clusters have been generated and updated at a previous time. If so, then the old values are updated using the FCM algorithm, and the validity index is evaluated. 


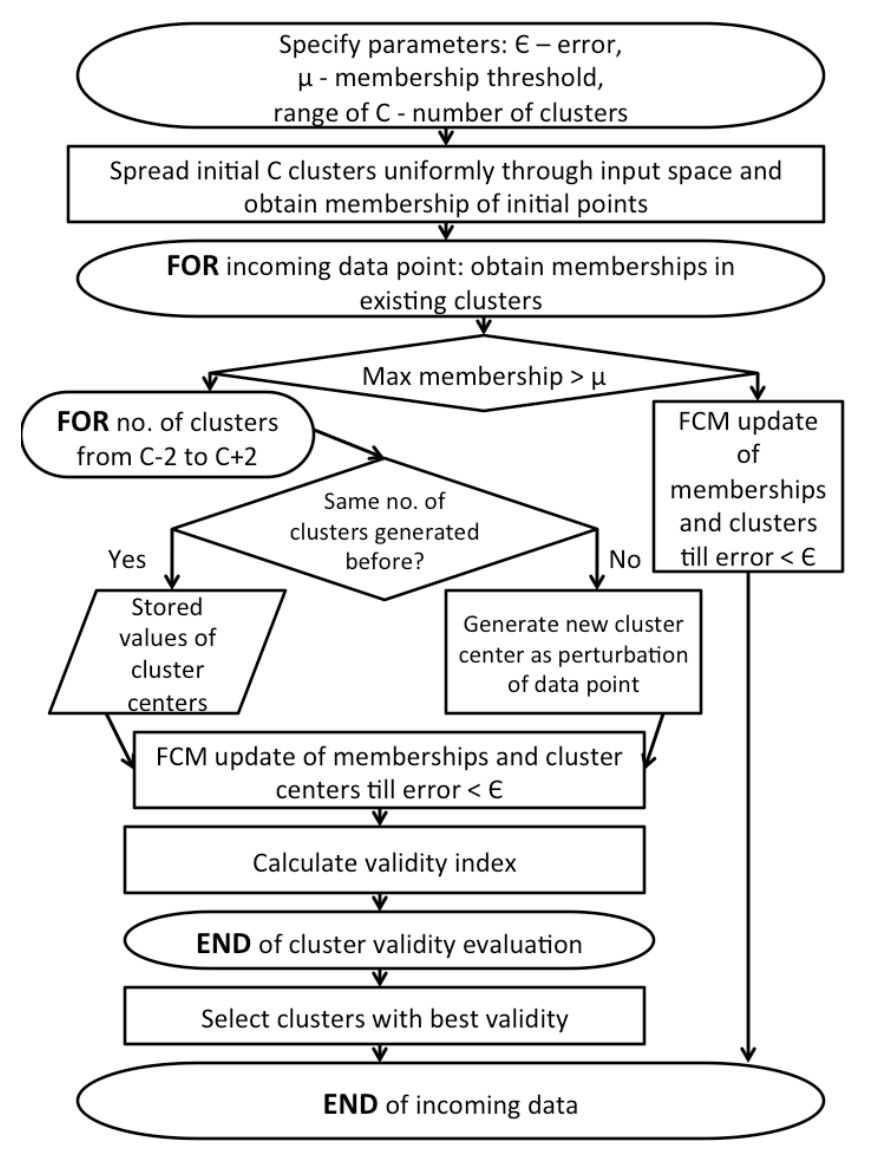

Figure 2: The flow chart of the dynamic FCM (dFCM) algorithm.

- If $L$ cluster centers have not been generated before, then the stored values of $L-1$ centers are used, and an $L^{\text {th }}$ center is generated by slightly perturbing the data point. The AO update is carried out, and the validity index is evaluated.

- The cluster centers that generate the best value of the validity index are taken.

5. The process continues until the data points stop streaming in.

\subsection{The Membership Threshold $(\mu)$ :}

The purpose of the membership threshold, $\mu$, is to avoid evaluating cluster validity each time a data point comes in. That is, if the data point lies to a specified satisfactory extent in a cluster, then it is not necessary to check if other clusters are better. However, the role of $\mu$ may be allowed to change, depending on what the specific application of the $\mathrm{dFCM}$ algorithm may require. The experimenter is free to set the conditions that need to be met in order to evaluate cluster validity. For instance, the condition that is specified in this work is whether or not the data point lies within a cluster to a satisfactory extent. In another application, it may be that validity index evaluation need only take place if the new updated centers are significantly different from the old ones. The factor $\mu$ can then be used in a condition that may look like:

$$
\left\|V_{\text {old }}-V_{\text {new }}\right\|>\mu,
$$

i.e., if the distance between the two sets of coordinates is greater than $\mu$, then validity is evaluated. In a situation like this, the $\mathrm{AO}$ update would take place automatically once a data point streams in, without any check on its membership. Once the centers are updated, $\mu$ can be used to check whether they are significantly different from the previous ones, and therefore whether or not validity should be evaluated. This prevents redundant calculations. 


\subsection{Cluster Centers and Validity}

Cluster centers are kept track of because validity indices may change as data points keep streaming in. At a certain point in time, two cluster centers may be sufficient, whereas at a later point, the algorithm may call for four cluster centers. The reverse may be true as well, and so, in order to keep the window open for cluster number possibilities, $C-2$ to $C+2$ clusters are checked, where $C$ is the actual number of cluster centers at a particular point in time. This window is flexible, and may be modified to be wider or narrower, depending on the preference of the experimenter. Note that the values of the cluster centers are made to fall within the initially specified range. However, the maximum number of cluster centers need not be specified if the experimenter prefers to keep the upper limit open. Any validity index favored by the experimenter may be employed, as the algorithm does not specifically depend on the type of validity index used.

\subsection{Running Time of $d F C M$}

The running time for the dFCM algorithm coupled with the Xie-Beni index was calculated and found to be $O\left(a n^{2}\right)$, where $n$ is the size of the data, and $a$ is the maximum number of AO updates required, explained below. The worstcase scenario was taken, in which a new cluster is generated for every incoming point, and there are no clusters to begin with. The running time had been obtained as follows: Simple arithmetic and logical operations take constant amounts of time. Unless a calculation that uses these operations explicitly depends on the size of the data or on a search, then the calculation also takes a constant amount of time. As each data point streams in, a check is performed to see if it lies within the established clusters to a satisfactory extent (step 3). Since we're assuming that each new point acts as a new cluster, each possible 'check' with the present clusters (i.e. in this case, the data points themselves) takes place. When the first point streams in, no check is performed, as there are no data points present. When the second point streams in, only 1 check is performed. When the next point streams in, 2 checks are performed, and so on. The total number of checks is represented by the following series:

$$
1+2+3+\cdots+n-1=\frac{n(n-1)}{2} .
$$

This implies a running time of order $O\left(n^{2}\right)$ for the various checks. When a new cluster is generated, validity indices are calculated for $L=C-2$ to $C+2$, and an $\mathrm{AO}$ update is performed (step 4).

The running time of the update depends on the number of data points already present, say $k$, and the number of iterations required before the error criteria is reached, say $a_{k+1}$, as it may differ for each case. The subscript $k+1$ indicates that the $(k+1)^{\text {th }}$ data point is streaming in. Therefore, the running time of the update can be considered to be $O\left(k a_{k+1}\right)$. The running time of the validity index calculations can be taken as a constant $(l=C+2-(C-2)=4)$ times $k$, as the validity index evaluation sums over all the data points present at the given time only once, and is performed an $l$ number of times. The total running time associated with each number contributing to Equation 8 can be thought of as: $O\left(k a_{k+1}\right)+O(l k)$ of which $O\left(k a_{k+1}\right)$ dominates. Since the order of the total running time of the AO updates can be thought of as:

$$
1 \cdot a_{2}+2 \cdot a_{3}+3 \cdot a_{4}+\cdots+(n-1) \cdot a_{n}
$$

where we define, $a=\max \left(a_{2}, a_{3}, \cdots, a_{n}\right)$. Therefore, the total running time of the dFCM algorithm can be considered to be: $O\left(a n^{2}\right)$.

\subsection{Applications of $d F C M$}

The dynamic fuzzy c-means clustering technique can be applied in situations that involve online analysis of streaming data, in which adaptive information is required, or in which the data to be clustered is not uniform. Most dynamic versions of available clustering techniques are application specific [22, 23]. However, dFCM is a generic algorithm that can be applied to a number of different situations. For instance, [14] discusses a potential application as an adaptive rule extraction technique for fuzzy associative memories, in the field of soft computing. In this paper, dFCM has been applied to high energy particle physics data reconstruction. Another potential application may lie in time-series analysis and prediction, or updating databases. 


\section{Calorimeter Concept and Design}

As a demonstration of the applicability of the FCM and DFCM clustering techniques, a sampling calorimeter consisting of tungsten and silicon layers was simulated using the GEANT4 package [24, 25]. A similar tungstensilicon calorimeter has been developed and used by the CALICE Collaboration [26, 27]. A sampling calorimeter consists of several planes of absorber material along with active medium planes. A design of a tungsten-silicon calorimeter was made with 20 layers, where each layer consisted of a $3 \mathrm{~mm}$ thick tungsten plate followed by a $0.3 \mathrm{~mm}$ thick silicon sensor. Being a high-Z element, tungsten converts high energy photons or electrons into electromagnetic showers. The majority of the photons that are emitted in high energy collisions are decay photons from $\pi^{0}$. One of the major goals is to reconstruct $\pi^{0}$ and their energy from the measured photon showers. The decay angle of the two emitted photons decreases with the increase of the energy of $\pi^{0}$. The distance between the two emitted photons has been calculated for a placement of the calorimeter at a distance of $350 \mathrm{~cm}$ from the interaction vertex, as shown in Figure 3 As the $\pi^{0}$ energy increases, the distance between the two photons decreases. The reconstruction of the photon showers needs to be accurate in order to obtain the shower positions of the photons and deposited energy. In order to measure $\pi^{0}$ energy accurately, tracking of the shower in different layers is needed. Therefore, the position resolution of the detectors in the sensitive medium has to be suitable.

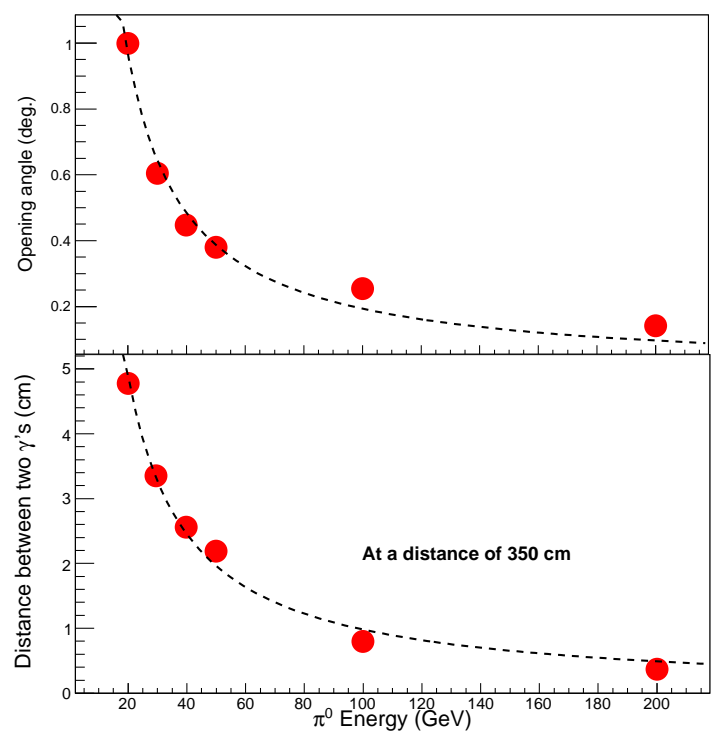

Figure 3: Opening angle (top panel) between two photons emitted from the decay of $\pi^{0}$ as a function of $\pi^{0}$ energy. The minimum distance between two photons at a distance of $350 \mathrm{~cm}$ from the vertex is shown in the bottom panel. As the $\pi^{0}$ energy increases, both the opening angle of the two decaying photons and the distance between them decrease. The dashed curves are fits to the data points, which agree with theoretically calculated values.

The calorimeter, shown in Figure 4 was designed keeping all of the requirements in mind. The longitudinal shower profile of the calorimeter, as shown in Figure 5 was studied in order to decide the granularity of the detector layers. From this figure, it was found that the maximum of the shower in terms of energy deposition occurs around layer 4 for photon energy of around $5 \mathrm{GeV}$, whereas for a photon of $50 \mathrm{GeV}$, the shower maximum is around layer 8. Therefore, high granular planes were placed in the region of the shower maxima for accurate measurements. For the purpose of simulation, only twenty layers were considered, out of which three layers $(4,8$ and 12) were made of highly granular silicon pads, each with dimension of $0.1 \mathrm{~cm} \times 0.1 \mathrm{~cm}$. The rest of the layers were made up of $1 \mathrm{~cm} \times 1 \mathrm{~cm}$ silicon pad detectors. The three high granular planes help to determine the shower position with high accuracy and thus help in tracking the path of the incoming particles. The shower generation, energy deposition and the characteristics of the shower produced by photons have been studied for the calorimeter using both FCM and dFCM techniques. 


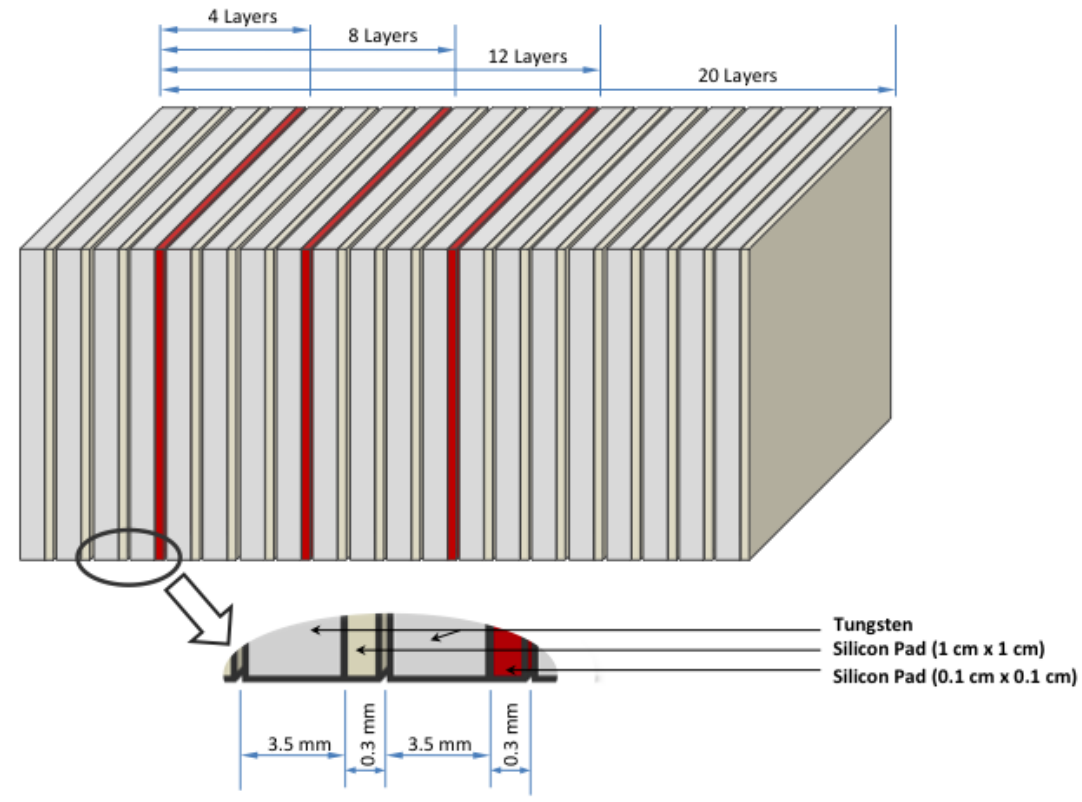

Figure 4: The components of a tungsten-silicon sampling calorimeter consisting of 20 layers of detectors. The three highlighted layers are made of highly granular $0.1 \mathrm{~cm} \times 0.1 \mathrm{~cm}$ silicon pads, and the rest of the layers consist of $1 \mathrm{~cm} \mathrm{x} 1 \mathrm{~cm}$ silicon pads.

\section{FCM applied to photon clusters}

In order to demonstrate the practicability of the FCM algorithm described in Section 2 , data for a single $\pi^{0}$ decay into two photon clusters at different energies was generated. Figure 6 displays the shower profiles of photons emitted from $\pi^{0}$ at three different energies: $10 \mathrm{GeV}, 50 \mathrm{GeV}$, and $100 \mathrm{GeV}$. The left column shows the event display of the three cases. The two photon showers move closer to one another with the increase of $\pi^{0}$ energy, thereby causing an overlap of clusters. The FCM algorithm was used to cluster the data points on each plane of the detector, taking the $x$ -position, $y$ - position and the energy depositions of each pad into account. The fuzzy factor $m$ was varied, and a value of 1.8 was found to be suitable in resolving the individual clusters. This value has been also been recommended in [3]. For all FCM clustering performed, the following parameter values were taken:

$$
m=1.8 \quad \text { and } \quad \epsilon=0.01 \text {. }
$$

The results of the clustering for layer 8 in terms of lateral shower profiles are shown in the right column of Figure 6 . The solid dots in the left column indicate the cluster centers found on each of the three planes. The lines represent the photon tracks. It is seen that despite the large extent of overlap, the photon clusters are clearly identified by the FCM algorithm, and the photon paths are successfully tracked using the three layers. The cluster positions, the tracks and the energy depositions of the photons have been used to reconstruct the mass of the $\pi^{0}$. This is a test of the working of the reconstruction algorithm, where clustering of the hit pads plays an important role. Figure 7 (a-c) shows the invariant mass reconstruction using the FCM algorithm, which essentially indicates the quality of $\pi^{0}$ reconsturction for three different energies. Figure 7 (d) shows the $\pi^{0}$ mass, reconstructed from the decay of photons for $\pi^{0}$ of different energies. The statistical errors indicated on this figure are the $r m s$ values of the invariant mass distributions. The granularity of the detector and the limitations of the clustering routine limit reconstruction of $\pi^{0}$. As seen from Figure 7(d), up to $100 \mathrm{GeV}$ energy, there is a reasomable mass reconstruction, beyond which it deviates. 


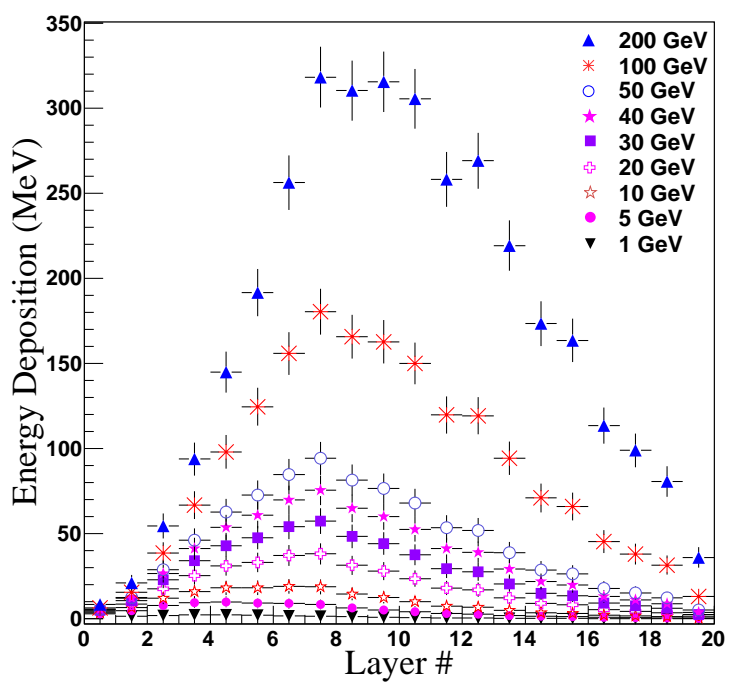

Figure 5: The longitudinal shower profile for energy deposition by photons of different energies in various layers of the sampling calorimeter.

\section{Application of dFCM to Calorimetric Data}

The dFCM clustering technique as discussed in Section 3 works equally well for the calorimetric data discussed in the previous section. Since FCM is a static algorithm that takes all data points into account at one time, it is sometimes unable to identify clusters with non-uniform data patterns, like those encountered in a real experimental scenario where a variety of particles hit the calorimeter. The dynamic version of the FCM algorithm works better to resolve very close clusters.

In order to determine the spatial resolving power of both FCM and AFCM, a sample of eight clusters on the fourth layer of the calorimeter was generated. Figure 8 shows the profiles of the eight clusters. Each data point represents the position and energy deposition on each of the small pads $(0.1 \mathrm{~cm} \times 0.1 \mathrm{~cm})$. First, the FCM clustering technique was performed, with an input cluster range of 2 to 10. The Xie-Beni index was used to select the best set of cluster centers. The minimum value of the index was obtained for two cluster centers, as the data appears to be distributed in two major groups of four clusters each. The solid points of the left panel of Figure 8 shows the two cluster centers obtained using the FCM algorithm and the Xie-Beni index on the raw data. The FCM clustering could not resolve the individual clusters, as they appear to be within a group.

Next, the dFCM algorithm was applied to the same data, and the Xie-Beni index was used to find the number of clusters. For all dFCM clustering performed, the following parameter values were taken:

$$
m=1.8, \quad \epsilon=0.01, \quad \mu=0.8, \quad \text { Number of initial points }=10 .
$$

The right panel of Figure 8 shows the result of clustering using the dFCM algorithm. As can be seen, there are some isolated scattered data points that affected the number of clusters obtained. The energy depositions of the scattered data points were low, and below that of one MIP (minimum ionizing particle). Thus, a cut of one MIP on a cell level was applied so that scattered points were not considered before clustering. With this cut, we observe that the dFCM method was able to resolve and identify all the eight individual clusters. This was possible because the dFCM method, as was emphasized earlier, allows clustering to be performed on various stages of data as the data points stream in, and adapts to the structure of data at each instant. The dFCM algorithm is more suitable to resolve clusters that are not uniformly distributed.

In order to demonstrate the power of the AFCM algorithm, around 1000 events of eight clusters were synthesized with varying strengths as well as positions. For each event, the dFCM algorithm was applied. In Figure 9 the output 


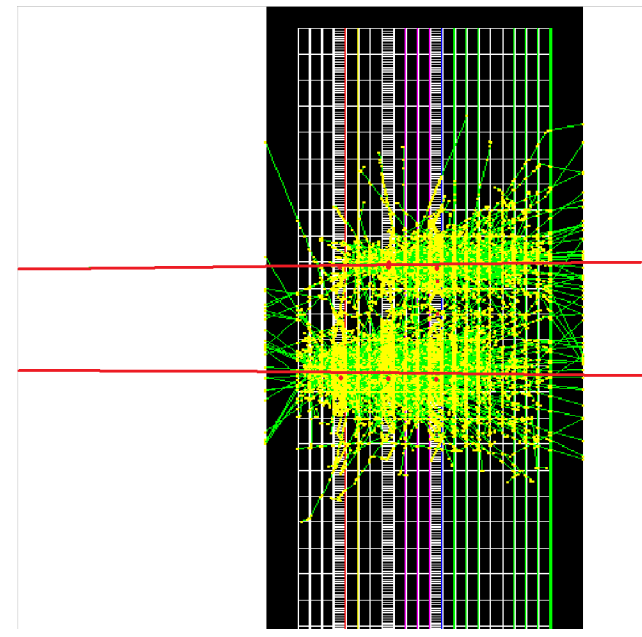

(a)

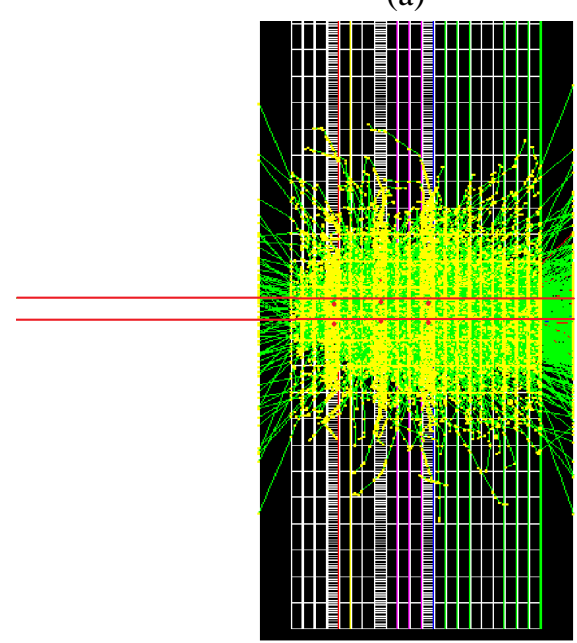

(c)

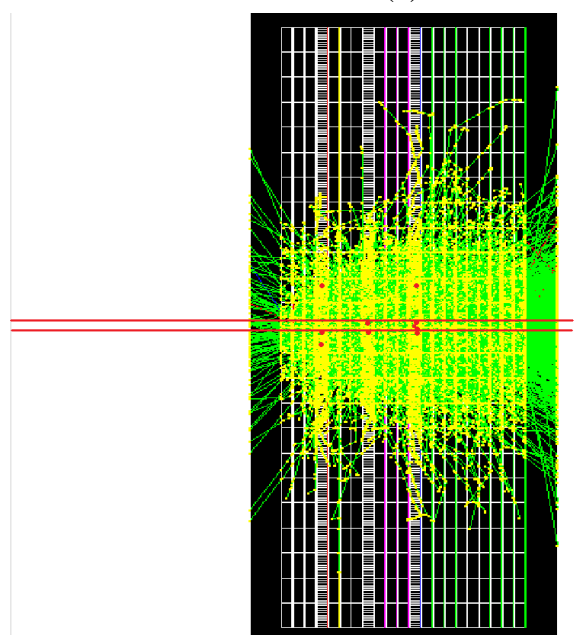

(e)

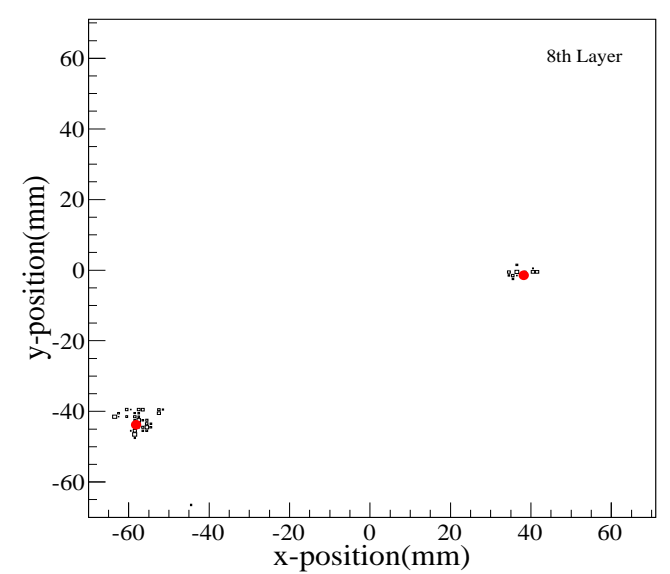

(b)

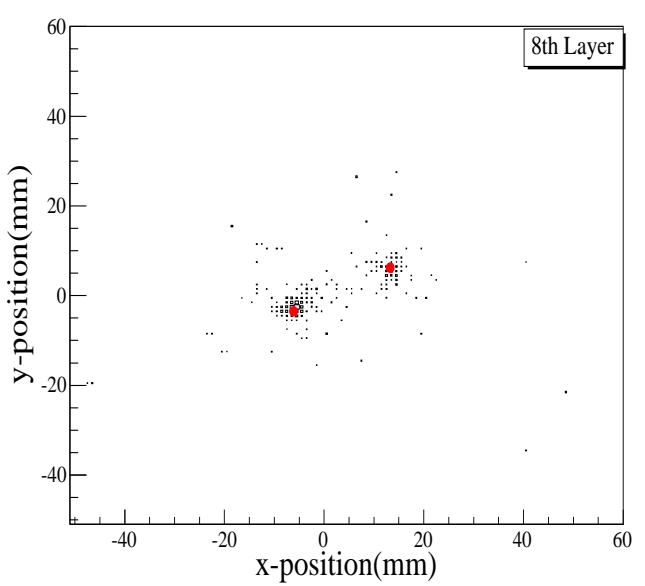

(d)

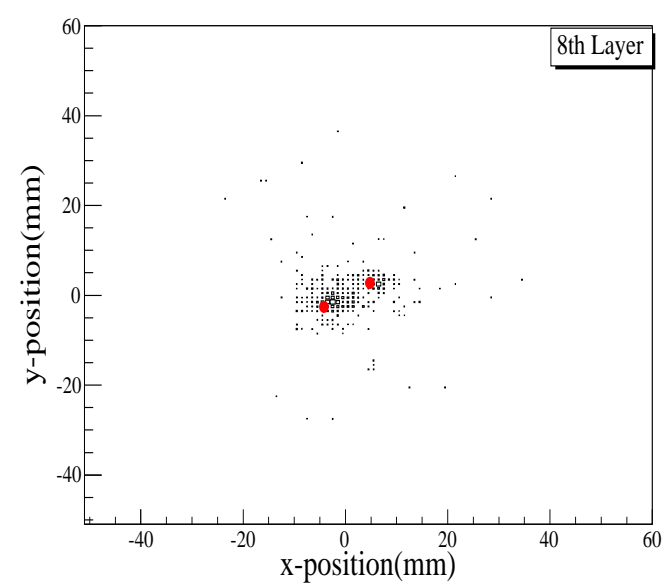

(f)

Figure 6: Profiles of neutral pions of energies $10 \mathrm{GeV}$ (a-b), $50 \mathrm{GeV}$ (c-d), and $100 \mathrm{GeV}$ (e-f) decaying to two photons. Left: Longitudinal shower profiles and the cluster centers found by the FCM algorithm on the 4th, 8th and 12th layers. Right: the lateral shower profiles of the 8th layer and the clusters found by the FCM algorithm. 


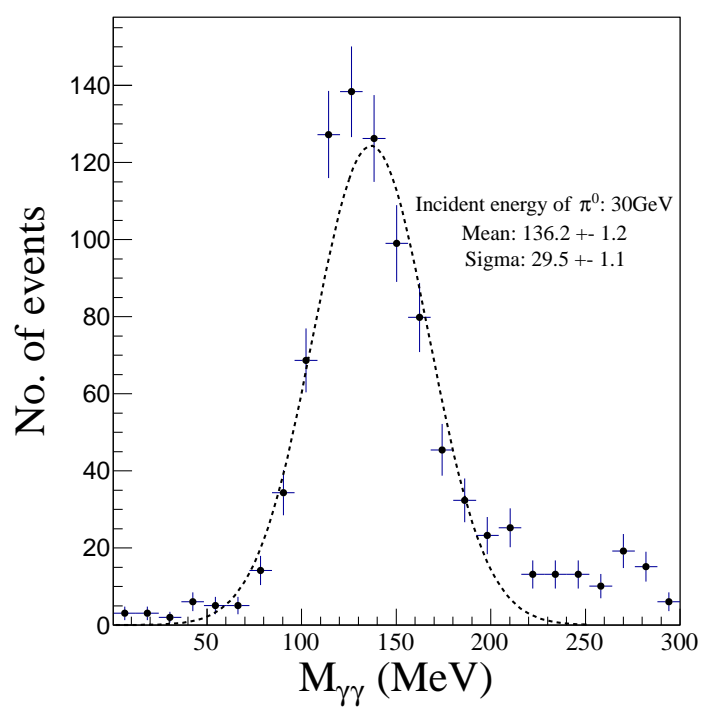

(a)

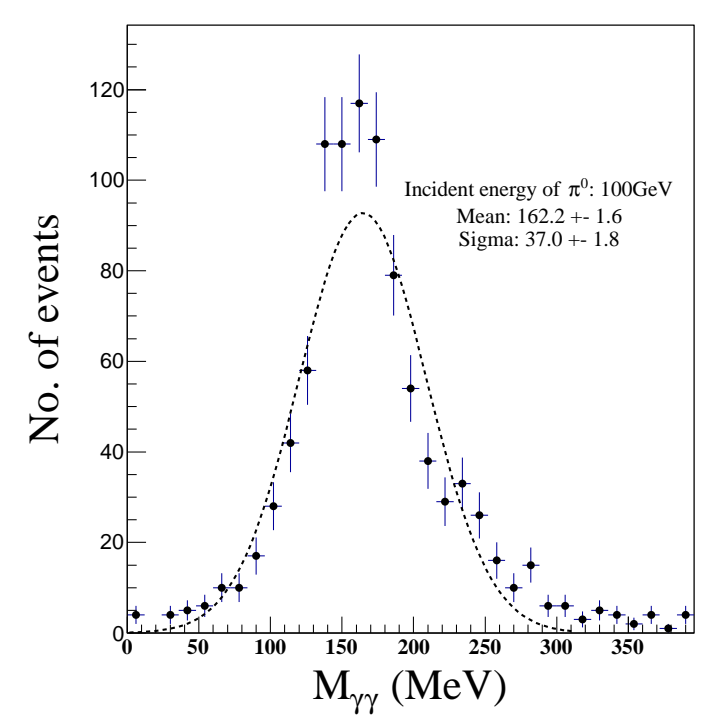

(c)

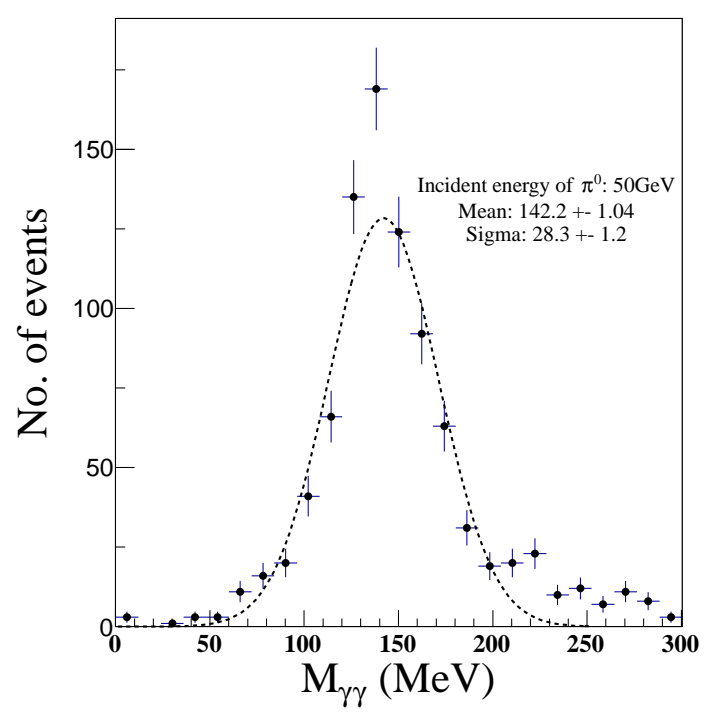

(b)

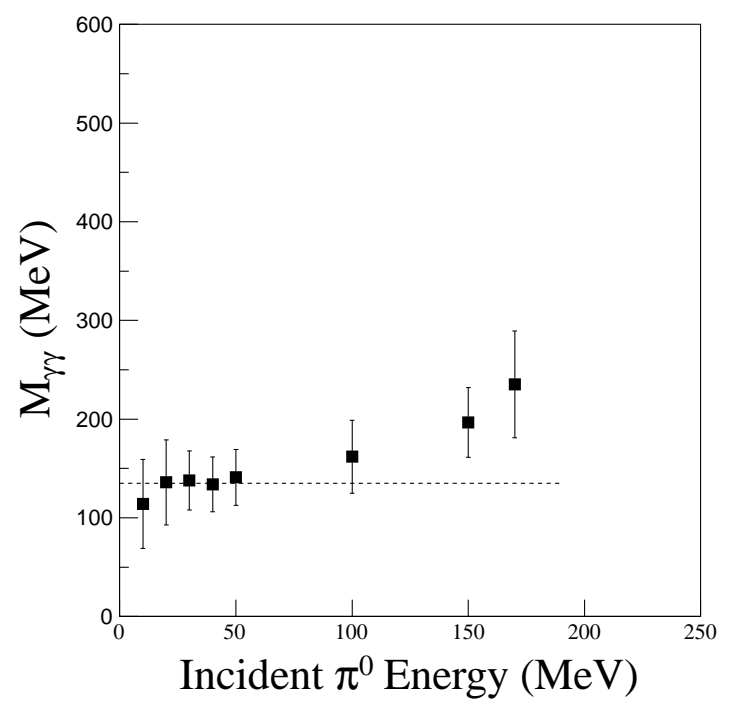

(d)

Figure 7: Invariant mass distributions of two photons in the sampling calorimeter using FCM clustering routine for $\pi^{0}$ energies of: (a) 30 GeV, (b) $50 \mathrm{GeV}$, (c) $100 \mathrm{GeV}$, and (d) Reconstructed $\pi^{0}$ mass by the measurement of cluster positions using FCM. Statistical errors are indicated in the figure. 


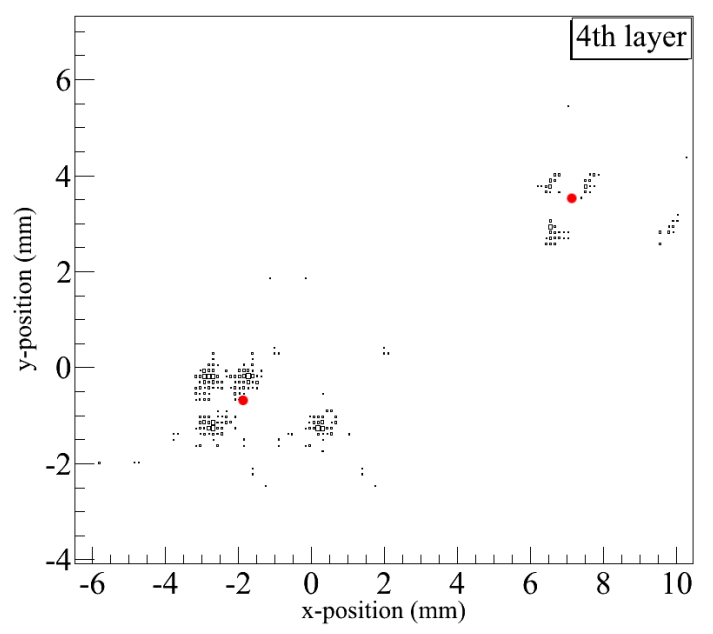

(a)

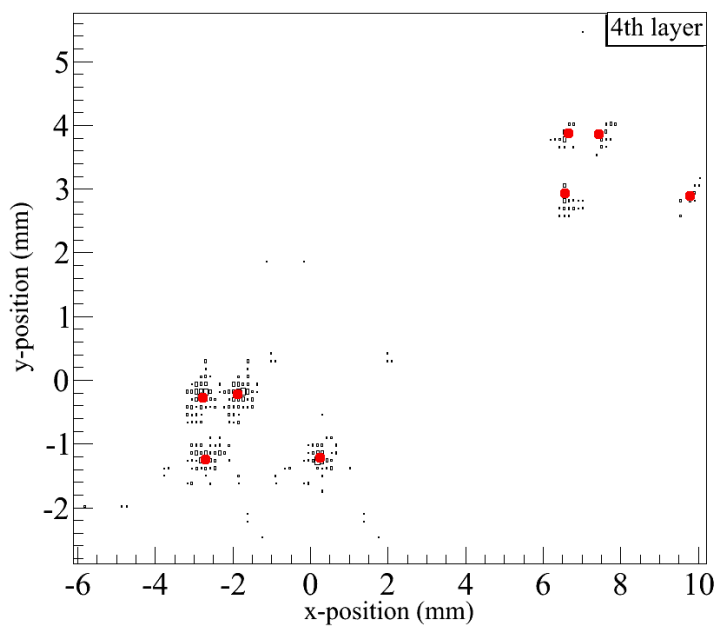

(b)

Figure 8: (a) Raw data points from the fourth layer of the calorimeter and the clusters found by using the simple FCM algorithm. The minimum of Xie-Beni validity index was obtained for the two cluster centers shown. (b) The results of clustering obtained by using the dFCM algorithm. All the clusters are properly accounted for.

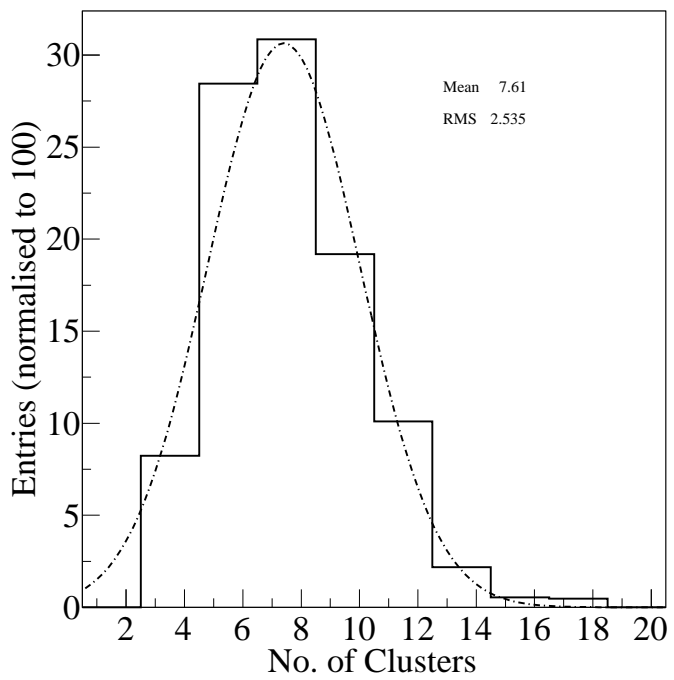

Figure 9: Number of clusters identified by the dFCM algorithm for known sets of eight clusters. The histogram is normalized to 100 clusters. A peak is observed around 8 clusters, and within \pm 1 , a total of 80 clusters can be accounted for. 
of the dFCM clustering in terms of number of identified clusters has been plotted for the case of eight known clusters. The histrogram is normalized to 100 . A peak is observed around 8 clusters, and within \pm 1 , a total of 80 clusters can be accounted for. That means for known 8 clusters, about $80 \%$ of the clusters can be found with number of clusters between 7-9. This tells about the quality of the clustering.

A note on the validity index: In order for a validity index to accurately select a set of cluster centers that represent a set of clusters well, a clustering algorithm must be capable of finding the clusters in the first place. That is to say, the clustering algorithm must find cluster center coordinates in such a way that minimizes the validity index that will ultimately be used to select the best set of coordinates. Even though the FCM algorithm performed clustering from 2 to 10 cluster centers, the coordinates that it found when it searched for 8 cluster centers did not eventually yield the minimal value of the Xie-Beni index. The index selected the better representative of the data to be 2 cluster centers. However, when the same index was used to select the best set of clusters found by dFCM, the minimal value was obtained when all 8 individual clusters were resolved.

While it is true that validity indices are by no means generic, it is safe to say that dFCM can at least find suitable clusters centers when searching over a range of them, whether or not the validity index can identify them. This is also true for FCM, which can be forced to find a certain set of clusters by selecting a single number of clusters to find. Keeping the role of the validity index in mind, the same experiment was repeated with the partition coefficient described by Equation 4 and its modification, described by Equation 5 . With FCM, the two coefficients were maximized when 2 cluster centers were obtained, whereas the PC selected 7 cluster centers and the MPC 9 cluster centers, with dFCM. Even when the cluster center ranges were modified to [3,10], FCM did not provide cluster centers that resolved the clusters well, and the validity indices indicated that having only 3 cluster centers was the better option.

Finally, as an application of the clustering algorithm to calorimetric data, a simulation of two $50 \mathrm{GeV}$ neutral pions, placed close together, were studied for the layers 4,8 and 12 where higher resolution silicon pads are placed. The data points in Figure 10 show the hits on the 4th, 8th and 12th layers of the calorimeter. The four clusters can be seen to be reasonably close together. The dFCM clustering algorithm was carried out to identify the clusters. A cut off of $89 \mathrm{keV}$, corresponding to one MIP, was applied before the clustering. The left side panels of (a), (c) and (e) give the results of the clustering routine with only a threshold of one MIP, applied before clustering. It is seen that in all cases, the major clusters have been accounted for. Some scattered data points that have higher energy depositions give additional clusters, which are essentially outliers. It is found that the energy depositions and number of hit cells of the outlier clusters are much smaller compared to the four main clusters for each layer. Using these data sets, the behaviour of the outliers are studied. It turns out that most of the clusters in the outliers are made up of a few cells with low values of deposited energies. Thresholds on the number of cells per cluster and deposited energy can be used to eliminate the outlier clusters. The panels, (b), (d), and (f) on the right side of the figure show the results of clustering after the application of thresholds on the cell hits, which eliminate the outliers in each of the layers. In case of actual calorimetric data, the thresholds can be optimized and applied in order to obtain the photon clusters.

\section{Summary}

In order to reconstruct the physical information extracted from calorimeters and other particle detectors, a suitable clustering technique must be applied. The ability of fuzzy clustering to assign data points to more than one cluster at a time makes it a powerful tool when clusters overlap, as is often the case with large particle densities of high energy experiments. The FCM technique and its dynamic version, dFCM, have been evaluated to be used in calorimetric data reconstruction.

A tungsten-silicon sampling calorimeter of 20 radiation lengths was configured using the GEANT4 package. The calorimeter consisted of 20 layers, each with one radiation thick tungsten and 300 micron silicon pad detectors. Layers 4,8 and 12 consisted of high resolution silicon pads, with $0.1 \mathrm{~cm} \times 0.1 \mathrm{~cm}$ sized pixels. The remaining layers were silicon pad layers with cell dimensions of $1 \mathrm{~cm} \times 1 \mathrm{~cm}$. The fuzzy c-means (FCM) clustering technique was applied to all the layers for the two photon clusters obtained after the decay of a neutral pion. The study demonstrated how the clusters and photon tracks could be identified for different energies of neutral pions. The tracks are successfully identified by locating the clusters in the 3 high resolution pad layers, and the total energy of a given track is obtained by summing over clusters of all pad layers belonging to the track. This provides a method for successful $\pi^{0}$ reconstruction in the calorimeter. 


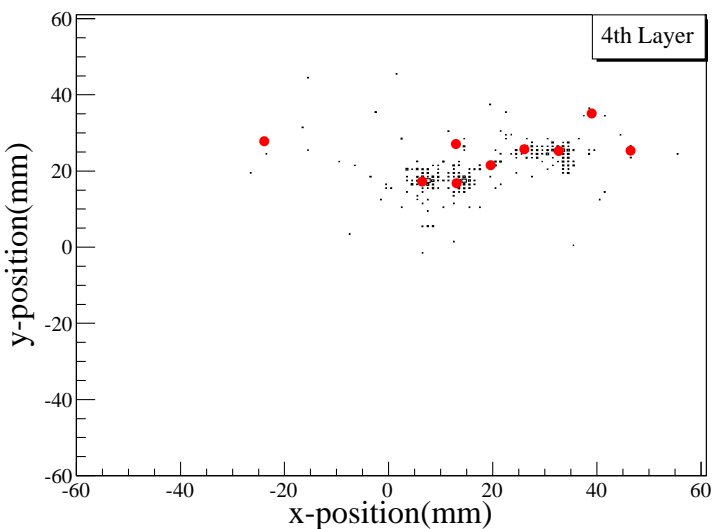

(a)

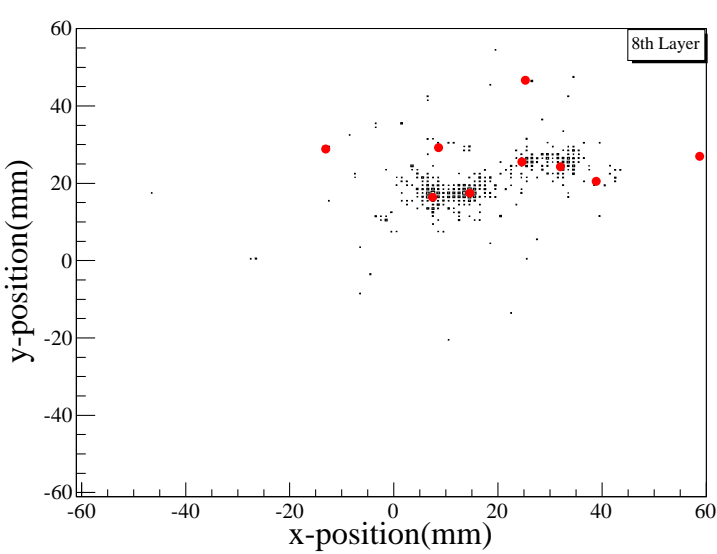

(c)

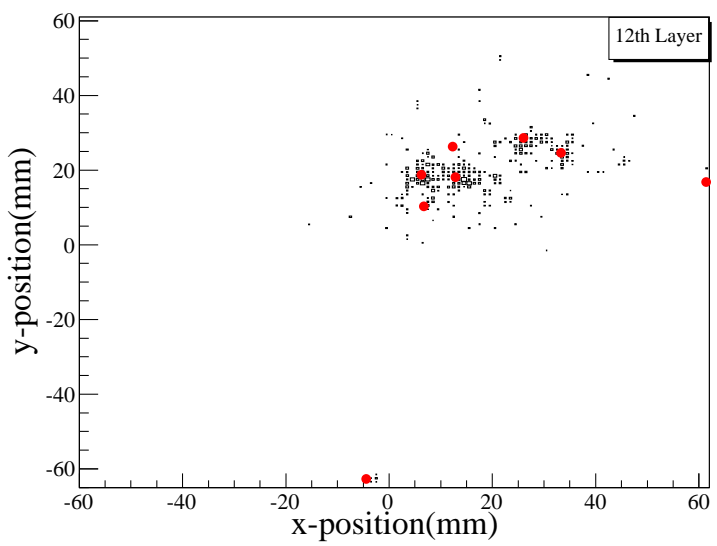

(e)

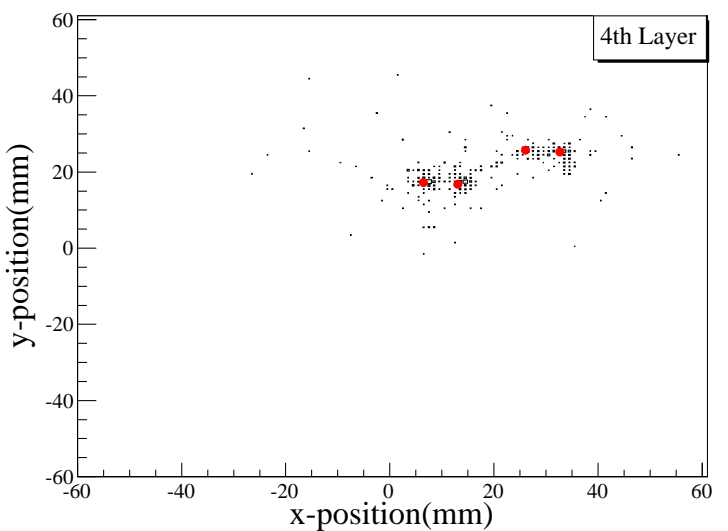

(b)

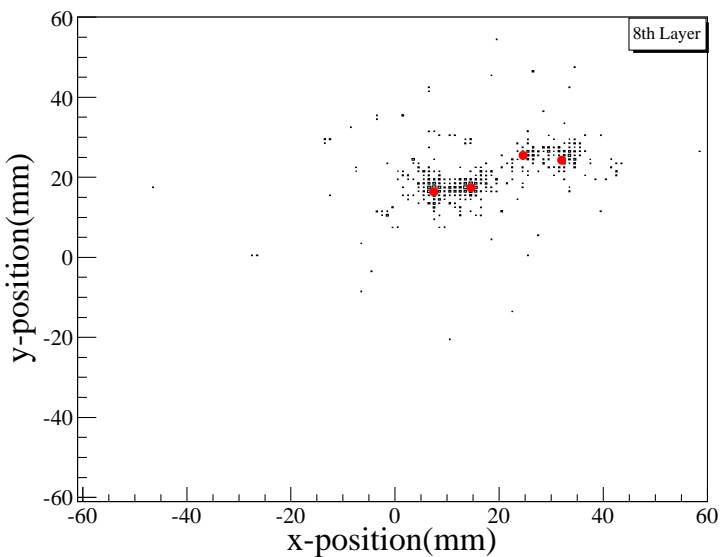

(d)

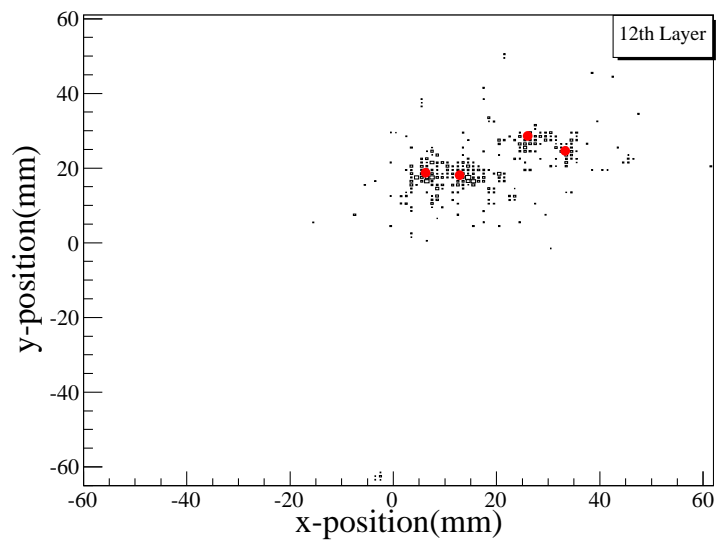

(f)

Figure 10: Hits on 4th, 8th and 12th layer of the calorimeter, obtained from two closely placed neutral pions, each of $50 \mathrm{GeV}$ energy. The left panels, (a), (c), and (e) show the results of the dFCM clustering routine, shown by the large solid points. The right panels, (b), (d) and (f) show the clusters which survive after a cut on the number of cell hits in a cluster. 
The dynamic version of the FCM algorithm (dFCM) allows clusters to be generated and eliminated as required as data streams in. The dFCM algorithm successfully identified the individual clusters in a non-uniformly distributed set of 8 photon clusters, whereas FCM was shown to consider a group of clusters to be one large cluster. The dFCM technique was also applied to a simulation of two $50 \mathrm{GeV}$ neutral pions decaying to two photons each. Each of the four photons were easily identified, despite the presence of small clusters of outliers. The energies and the number of points in each of the outlier clusters are usually small enough for a suitable elimination criteria to be defined. However, as different energies as well as different layers give rise to varying densities and different cluster structures, a meticulous study needs to be performed in order to come up with proper discrimination criteria. The discrimination criteria will have to be tuned in a realistic environment with different colliding systems and colliding energies.

\section{References}

[1] Lotfi A. Zadeh, Communications of the ACM, 37 No. 3 (1994) 77.

[2] Andries Engelbrecht in "Computational Intelligence: An Introduction", Wiley \& Sons. ISBN 0-470-84870-7 (2007).

[3] S.K. Pal, S. Chattopadhyay and Y.P. Viyogi, Nucl. Instr. and Meth. in Physics Research A626 (2011) 105.

[4] S. Chattopadhyaya, Z. Ahammed, Y.P. Viyogi, Nucl. Inst. and Meth. in Physics Researh A421 (1999) 558.

[5] M. Ambriolaa, R. Bellottia, M. Castellanoa, G. De Cataldo, C. De Marzo, Nucl. Inst. and Meth. in Physics Researh A387 (1997) 83.

[6] Shimon Whiteson and Daniel Whiteson, Engineering Applications of Artificial Intelligence, 22 (2009) 1203.

[7] U. Muller, Nucl. Instr. and Meth. in Physics Research A502 (2003) 811.

[8] J. Freeman, W. Ketchumb, J.D. Lewis, S. Poprocki, A. Pronko, V. Rusu, and P. Wittich, Nucl. Instr. and Meth. in Physics Research A663 (2012) 37.

[9] Mei-Ling Yu, Kun-Shi Zhang, Lian-Shou Liu, Int. Jour. Mod. Phys. A20 (2005) 7603.

[10] M. Mjahed, Nucl. Inst. Meth., 559 (2006) 199.

[11] R. Nock, F. Nielsen, IEEE Trans. on Pattern Analysis and Machine Inteligence, 28 (2006) 1.

[12] J. C. Bezdek, "Pattern Recognition with Fuzzy Objective Function Algorithms", New York: Plenum. ISBN 0-306-40671-3 (1981)

[13] N.R. Pal and J. C. Bezdek, IEEE Trans. Fuzzy Systems, 3 No. 3 (1995) 370.

[14] R.P. Sandhir, S.Kumar, Proc. IEEE International Conference on Fuzzy Systems, (2010) 1.

[15] G. Suliman, D. Bucurescu, Romanian Reports in Physics, 62 No. 1 (2009) 27.

[16] X.L. Xie and G. Beni, IEEE Trans. Pattern Analysis and Machine Intelligence, 13 No. 8 (1991) 841

[17] R.N. Dave, Pattern Recognition Lett., 17 (1996) 613.

[18] D.S.Yeung and X.Z.Wang, IEEE Trans. Pattern Analysis and Machine Intelligence, vol. 24 No. 4 (1992) 556.

[19] X. Wang, Y. Wang, and L. Wang, Pattern Recognition Letters, 25 No. 10 (2004) 1123.

[20] H. Choe and J.B. Jordan, Proc. IEEE International Conference on Fuzzy Systems, (1992) 349.

[21] J. Fan, W. Zhen, and W. Xie, Pattern Recognition Letters, 24 (2003) 1607.

[22] Ming, Yu, A. Malvankar, and Li Yan, Proc. IEEE International Conference on Networks, (2005)

[23] F. Ensan, M.H. Yaghmaee and E. Bagheri, Proc. 11th IEEE Symposium on Computers and Communications, (2006) 442.

[24] S. Agostinelli et al., Nucl. Inst. and Meth., A506 (2003) 250

[25] J. Allison et al. IEEE Trans. on Nucl. Sci. 53 (2006) 270.

[26] Daniel Jeans et al., CALICE Collaboration, Nucl. Inst. Method. 628 (2011) 324.

[27] F.Salvatore et al., CALICE Collaboration, Nucl. Inst. Method. 608 (2009) 372. 